\title{
MÉTODOS DE FORÇAMENTO DE BORBULHAS E APLICAÇÃO DE CIANAMIDA HIDROGENADA PARA PRODUÇÃO DE MUDAS DE LARANJA 'VALÊNCIA' SOBRE CITRUMELO 'SWINGLE' EM VIVEIRO TELADO'
}

\author{
BRUNO FERNANDO FARIA PEREIRA² \& SÉRGIO ALVES DE CARVALHO ${ }^{3}$
}

\begin{abstract}
RESUMO - O objetivo deste experimento foi avaliar diferentes métodos para o forçamento de borbulhas de laranja 'Valência' enxertada em citrumelo 'Swingle': vergamento e decapitação, associados à aplicação de cianamida hidrogenada. Utilizando-se do delineamento experimental inteiramente casualizado, em esquema fatorial 2 x 2, avaliaram-se o curvamento ou a decapitação da haste do porta-enxerto, e a aplicação ou não do regulador de crescimento à base de cianamida hidrogenada 1\% (C.H.). Avaliou-se o índice de brotação das borbulhas aos 50 e 80 dias, bem como o comprimento, diâmetro da haste e número de folhas aos 80; 110 e 140 dias após a aplicação dos tratamentos. A C.H. não influenciou significativamente na brotação de borbulhas. A decapitação elevou a brotação em $26 \%$ em relação ao vergamento. Por outro lado, o vergamento da haste do porta-enxerto proporcionou maior vigor às plantas, considerando-se comprimento, diâmetro e número de folhas das hastes. Na avaliação final, as plantas submetidas ao vergamento apresentavam altura de haste $75 \%$ maior do que as submetidas ao forçamento por decapitação.
\end{abstract}

Termos para indexação: propagação, enxertia, vergamento, decapitação, cianamida hidrogenada.

\section{BUD-FORCING METHODS FOR 'VALENCIA' ORANGE AND HIDROGEN CYANAMIDE APPLICATION ON 'SWINGLE' CITRUMELO NURSERY-TREES PRODUCTION UNDER SCREEN}

\begin{abstract}
The objective of this research was to evaluate differents citrus bud-forcing methods of 'Valência' orange grafted on 'Swingle' citrumelo: bending and cutting off the rootstock stem, associated with hydrogen cyanamide application. Using a completely randomized experimental design in a $2 \times 2$ factorial it was evaluated the bending or the cutting off rootstock stem, and the application or not of the growth regulator in hydrogen cyanamide base $1 \%$ (H.C.). It was evaluated the bud sprout index at the $50^{\text {th }}$ and the $80^{\text {th }}$ days, as well the height, stem diameter and the number of leaves at the $80^{\text {th }}, 110^{\text {th }}$ and $140^{\text {th }}$ days after the treatments aplication. The H.C. did not influence significantly on bud sprout. The cutting off rised the bud sprout in $26 \%$ in relation to bending. On the other hand, bending the rootstock stem provided the largest vigour to the plants, considering the stem height, diameter and number of leaves of the stems. In the final evaluation, the plants forced by bending showed stem height $75 \%$ higher than the cutting off treatment.
\end{abstract}

Index terms: propagation, grafting, bending, cutting off, hydrogen cyanamide.

Nos últimos anos, tem ocorrido um grande interesse no citrumelo 'Swingle' (Citrus paradisi Macf $x$ P. trifoliata Raf) para produção de mudas cítricas no Estado de São Paulo, com aumento de 20\% no número de mudas produzidas sobre este porta-enxerto no período de 1999 e 2003 (Pompeu Júnior et al., 2004). Comportamento semelhante foi observado no Estado da Flórida-EUA, entre os anos de 1983 e 1993, tendo esta variedade um crescimento de $15 \%$ para $51 \%$, considerando todas as outras variedades utilizadas (Williamson \& Maust, 1996). Dentre as características vantajosas desse porta-enxerto, destacam-se a excelente qualidade de frutos induzida à copa e, nas condições brasileiras, sua resistência à morte súbita dos citros (MSC), doença de causa ainda não determinada (Barbasso, 2005; Gimenes-Fernandes et al., 2001; Pompeu Júnior, 1999).

Embora o citrumelo 'Swingle' possua diversas características desejáveis, alguns viveiristas têm encontrado dificuldades no pegamento de borbulhas nele enxertadas e no maior tempo necessário para formação de mudas. A rápida e uniforme brotação da borbulha, e a redução no período de formação favorecem o menor custo de produção e disponibilidade de mudas em todas as épocas propícias para a comercialização e plantio (Coelho et al., 1983).

O forçamento da borbulha na produção de mudas em viveiros de campo pode ser feito por diferentes processos, como curvamento, anelamento, decapitação ou torção da haste do porta-enxerto. Diferenças em resultados obtidos em condições de clima tropical e subtropical, na eficiência dos métodos e momentos de forçamento de borbulhas, são discutidas por Samson \& Bink (1976), que demonstraram a atuação de reguladores vegetais, como auxinas, citocininas e carboidratos neste processo, com a avaliação do curvamento e da decapitação associados à aplicação de reguladores de crescimento. Segundo os autores, nos trópicos úmidos, a respiração é elevada o ano todo, possuindo a planta pouca reserva de carboidratos, sendo a decapitação um processo insatisfatório. Além disso, o curvamento seria um meio mais seletivo de estimular as gemas, já que o transporte de auxina continua intacto em um lado do ramo, enquanto a dominância apical é quebrada somente no local acima da inserção da borbulha.

Em viveiro de campo, Carvalho (1992) comparou quatro métodos de forçamento aplicados na combinação laranja 'Pêra' sobre limão 'Cravo'. Esse autor observou que a decapitação foi mais eficiente na indução da brotação embora tenha proporcionado menor velocidade no crescimento das mudas. Por outro lado, curvamento foi o método que proporcionou maior crescimento e vigor para as mudas, embora tenha sido menos eficiente na brotação. Bowman (1999), em OrlandoFL, comparando vergamento e decapitação, relatou ter obtido maior vigor, rapidez na brotação e sobrevivência dos brotos, empregando o primeiro processo para citrumelo 'Swingle', citrange 'Carrizo' [Citrus sinensis (L.) Osbeck x P. trifoliata Raf] e outros 17 híbridos.

Diversos trabalhos na área citrícola discorrem sobre o uso de reguladores vegetais relacionando-os principalmente com o aumento do vigor da vegetação, redução da florada, fixação, desbaste e aumento do tamanho dos frutos e atraso da colheita (Castro et al., 1996; Schafer et al., 2001; Cañizares \& Rojas, 2001). A cianamida hidrogenada (C.H.) é um regulador de crescimento muito utilizado em regiões de clima temperado, em frutíferas como videiras, macieiras e figueiras, visando à quebra de dormência de gemas, estímulo e uniformização da brotação, ou até mesmo à antecipação da produção de plantas adultas. Geralmente é aplicada em videiras, macieiras e figueiras (Mashima et al., 2000; Petri, 1997; Norberto et al., 2001; Mielle, 1991). Em citros, Cañizares \& Rojas (2001) observaram que os tratamentos com 1 e 1,5\% de C.H. incrementaram a vegetação e a florada da lima ácida 'Tahiti'. Não foram encontradas referências na literatura envolvendo a aplicação de C.H. na produção de mudas cítricas ou outros reguladores de crescimento aplicados diretamente na borbulha enxertada.

\footnotetext{
${ }^{1}$ (Trabalho 142/2005). Recebido: 05/09/2005. Aceito para publicação: 23/02/2006. Apoio: FAPESP e CNPq.

${ }^{2}$ MSc Eng ${ }^{\circ}$ Agrônomo, Instituto Agronômico - IAC, bolsista FAPESP. e-mail: brunoffp2000@yahoo.com.br.

3 Pesquisador Científico VI, Centro Avançado de Pesquisa Tecnológica do Agronegócio de Citros “Sylvio Moreira”. Caixa Postal 04 - CEP 13490-970. CordeirópolisSP. Bolsita do CNPq. e-mail: sergio@centrodecitricultura.br.
} 
Este trabalho foi realizado com o objetivo de avaliar a decapitação e o vergamento da haste do porta-enxerto associados ou não à aplicação da cianamida hidrogenada, no forçamento de borbulhas de laranja 'Valência' enxertada sobre citrumelo 'Swingle' em viveiro telado.

O experimento foi conduzido no Centro Avançado de Pesquisa Tecnológica do Agronegócio de Citros Sylvio Moreira do Instituto Agronômico (CAPTACSM-IAC), Cordeirópolis-SP, em viveiro com cobertura plástica e proteção lateral com tela antiafídeos, entre janeiro e setembro de 2001. Como porta-enxerto, utilizou-se o citrumelo 'Swingle' (Citrus paradisi Macf. x Poncirus trifoliata Raf), sendo as sementes retiradas de frutos maduros de plantas pertencentes ao Banco de Matrizes de porta-enxertos do CAPTACSM-IAC. Foram semeadas três sementes por tubete plástico cônico de $0,05 \mathrm{dm}^{3}$ com estrias e perfuração basal, preenchido por substrato comercial plantmax Citrus ${ }^{\circledR}$, à base de vermiculita, casca de pínus e húmus, suplementado com adubo de liberação lenta Osmocote ${ }^{\circledR}$ de formulação 19-6-10 (N, P, K).

Após a germinação, realizou-se o desbaste, deixando apenas uma planta por tubete, e 112 dias após a semeadura, as plantas, com aproximadamente $15 \mathrm{~cm}$ de comprimento, foram transplantadas para recipientes plásticos (citrovasos) com capacidade de $3,8 \mathrm{dm}^{3}$ utilizando substrato comercial rendmax Citrus $^{\circledR}$, à base de vermiculita, casca de pínus e húmus, acrescido de adubo de liberação lenta de formulação 22 4-8 (N, P, K), na proporção de $3 \mathrm{~g} \mathrm{~L}^{-1}$. Oitenta e quatro dias após o transplantio, foi realizada a enxertia a 15 centímetros de altura, pelo método "T" invertido, com borbulhas de laranja "Valência' [Citrus sinensis (L.) Osbeck] produzidas em borbulheira adensada sob telado da Unidade. Quatorze dias após a enxertia, realizou-se a aplicação dos tratamentos. Durante a fase de sementeira e viveiro, as plantas receberam os tratos culturais rotineiros para formação de mudas cítricas, conforme Carvalho (2001).

O delineamento experimental utilizado foi o inteiramente casualizado, em esquema fatorial $2 \times 2$, avaliando-se, no forçamento das borbulhas, a decapitação e o vergamento da haste do porta-enxerto e a aplicação ou não de regulador de crescimento à base de cianamida hidrogenada $\left(\right.$ Dormex $\left.^{\circledR}\right)$, na concentração de 1\%. Foram utilizadas 5 repetições, com 24 plantas por parcela, sendo 8 na área útil. $O$ vergamento foi realizado na porção da haste do porta-enxerto imediatamente acima do local da enxertia, aplicando-se uma torção e curvando-a para o lado oposto à inserção da borbulha, prendendo-se a parte superior da haste do porta-enxerto na sua própria base junto ao vaso. A decapitação foi feita através de poda em corte oblíquo às borbulhas, a 1 centímetro acima da enxertia, sendo o corte protegido com pasta cúprica. A aplicação da cianamida hidrogenada nas borbulhas foi feita com o auxílio de um pincel, após a retirada da fita plástica transparente biodegradável (20 dias após a enxertia). Realizaram-se avaliações da brotação das borbulhas aos 50 e 80 dias, bem como o comprimento, diâmetro da haste e número de folhas aos 80; 110 e 140 dias após a aplicação dos tratamentos. A cada duas semanas, realizaram-se desbrota lateral dos porta-enxertos e monda de plantas espontâneas.

Não ocorreram interações significativas entre a cianamida hidrogenada (C.H.) e os métodos de forçamento em nenhuma das avaliações realizadas.

Mesmo não havendo efeito significativo da aplicação de C.H. para a maioria dos parâmetros avaliados, nota-se, pela avaliação aos 50 dias, que o produto acelerou a brotação, proporcionando uma diferença de aproximadamente $15 \%$ no índice de brotação (Tabela 1). Não foram encontrados, na literatura, relatos envolvendo a aplicação de C.H. buscando precocidade e uniformização do estande na produção de mudas cítricas. Por outro lado, para espécies vegetais adultas, de regiões com clima temperado, principalmente uva, pêssego e macieira, diversos trabalhos relatam efeito positivo desse regulador de crescimento, antecipando e uniformizando a brotação e diminuindo a dominância apical (Miele, 1991; Petri, 1997; Marodin et al., 2002). A ausência de efeito significativo da C.H. na brotação de mudas cítricas, observada na presente pesquisa, provavelmente está associada às diferenças fisiológicas dos citros, espécie de região subtropical, em relação às espécies de regiões de clima temperado, para as quais a C.H. é amplamente recomendada. O mecanismo de atuação da C.H. na quebra de dormência ainda não foi totalmente elucidado, mesmo para espécies vegetais de regiões de clima temperado. Sabe-se que a aplicação da C.H. proporciona um aumento de peróxido de hidrogênio nos tecidos das gemas (Perez \& Burgos, 2004), o que pode ser responsável pela ativação do ciclo da pentose e conseqüente quebra de dormência das gemas (Omran, 1980).

A decapitação do porta-enxerto em relação ao vergamento acelerou a brotação das borbulhas na avaliação dos 50 e 80 dias em $26 \%$ e 7\%, respectivamente, em relação ao vergamento (Tabela 2). Os resultados obtidos aqui corroboram os obtidos por Carvalho (1992), que comparou quatro métodos de forçamento, incluindo a decapitação e o vergamento, na combinação laranja 'Pêra' sobre limão 'Cravo'. Este autor concluiu que a decapitação foi o tipo de forçamento mais eficiente para a indução da brotação. Concordam também com as informações obtidas por Williamson \& Maust (1996), na avaliação de métodos de forçamento para laranja 'Hamlin' sobre citrumelo 'Swingle', tendo obtido 100\% de brotação empregando a decapitação e apenas $41 \%$ utilizado o vergamento.

Aos 140 dias após a enxertia, as mudas apresentaram comprimento médio inferior a $50 \mathrm{~cm}$, considerado como adequado por Oliveira et al. (2005). Isso pode estar relacionado ao médio vigor do citrumelo 'Swingle' em viveiro e ainda à baixa temperatura média observada no período desta pesquisa, que foi de $23{ }^{\circ} \mathrm{C}$. Conforme Oliveira et al. (2005), a temperatura que maximiza a produção de mudas, está entre 26 e $28{ }^{\circ} \mathrm{C}$, ou seja, no mínimo $3{ }^{\circ} \mathrm{C}$ acima das condições observadas neste experimento.

Apesar da menor brotação, de maneira geral, o vergamento da haste do porta-enxeto a $5 \mathrm{~cm}$ acima da enxertia proporcionou maior vigor às plantas, considerando-se o número de folhas, o comprimento e

TABELA 1 - Porcentagem média de brotação aos 50 e 80 dias, e parâmetros de crescimento dos brotos, avaliados aos 80 ; 110 e 140 dias após a aplicação de diferentes doses de Cianamida Hidrogenada ${ }^{\circledast}$ nas borbulhas de laranja 'Valência' enxertadas em citrumelo 'Swingle'.

\begin{tabular}{|c|c|c|c|c|c|}
\hline \multirow{2}{*}{$N^{0}$ de dias } & \multirow{2}{*}{ Parâmetros } & \multicolumn{2}{|c|}{ Doses Cianamida Hidrogenada } & \multirow[b]{2}{*}{ D.M.S. } & \multirow[b]{2}{*}{ C.V. $(\%)$} \\
\hline & & $0 \%$ & $1 \%$ & & \\
\hline 50 & \% de Brotação & $61,87 \mathrm{a}$ & $72,5 \mathrm{a}$ & 25,22 & 35,70 \\
\hline \multirow{4}{*}{80} & \% de Brotação & $97,5 \mathrm{a}$ & $96,25 \mathrm{a}$ & 4,59 & 4,99 \\
\hline & $\mathrm{N}^{\circ}$ de folhas & $5,53 \mathrm{a}$ & $5,37 \mathrm{a}$ & 1,02 & 18,88 \\
\hline & Comprimento $(\mathrm{cm})$ & $3,83 \mathrm{a}$ & $3,89 \mathrm{a}$ & 1,38 & 37,77 \\
\hline & Diâmetro $(\mathrm{cm})^{(1)}$ & $0,83 \mathrm{a}$ & $0,84 \mathrm{a}$ & 0,01 & 2,16 \\
\hline \multirow{3}{*}{110} & $\mathrm{~N}^{\circ}$ de folhas & $7,35 \mathrm{a}$ & $8,13 \mathrm{a}$ & 1,27 & 17,34 \\
\hline & Comprimento $(\mathrm{cm})$ & $6,45 \mathrm{a}$ & $8,27 \mathrm{a}$ &, 18 & 31,34 \\
\hline & Diâmetro $(\mathrm{cm})^{(1)}$ & $0,87 \mathrm{a}$ & $0,88 \mathrm{a}$ & 0,01 & 2,15 \\
\hline \multirow{3}{*}{140} & $\mathrm{~N}^{\circ}$ de folhas & $12,69 \mathrm{a}$ & $12,85 \mathrm{a}$ & 1,35 & 11,21 \\
\hline & Comprimento $(\mathrm{cm})$ & $17,40 \mathrm{a}$ & $16,88 \mathrm{a}$ & 3,38 & 20,85 \\
\hline & Diâmetro $(\mathrm{cm})^{(1)}$ & $0,91 \mathrm{a}$ & $0,92 \mathrm{a}$ & 0,02 & 2,33 \\
\hline
\end{tabular}

Médias seguidas da mesma letra nas linhas não diferem entre si, pelo teste de Tukey, ao nível de 5\% de probabilidade.

${ }^{(1)}$ Médias transformadas em S.Q.R.T ( $\left.\mathrm{x}+\mathrm{a}\right) \mathrm{a}=0.5$. 
TABELA 2 - Porcentagem média de brotação aos 50 e 80 dias, e parâmetros de crescimento dos brotos, avaliados aos 80 ; 110 e 140 dias após a aplicação do vergamento ou decapitação na enxertia de laranja 'Valência' sobre citrumelo 'Swingle'.

\begin{tabular}{|c|c|c|c|c|c|}
\hline \multirow{2}{*}{$\mathbf{N}^{0}$ de dias } & \multirow{2}{*}{ Parâmetros } & \multicolumn{2}{|c|}{ Métodos de Forçamento } & \multirow{2}{*}{ D.M.S. } & \multirow{2}{*}{ C.V. } \\
\hline & & Vergamento & Decapitação & & \\
\hline 50 & \% de Brotação & $54,37 \quad b$ & $80,00 \mathrm{a}$ & 25,22 & 35,70 \\
\hline \multirow{4}{*}{80} & \% de Brotação & $93,75 \mathrm{~b}$ & $100,00 \mathrm{a}$ & 4,59 & 4,99 \\
\hline & $\mathrm{N}^{\circ}$ de folhas & $6,24 \mathrm{a}$ & $4,65 \mathrm{~b}$ & 1,02 & 18,88 \\
\hline & Comprimento $(\mathrm{cm})$ & $4,70 \mathrm{a}$ & $3,03 \mathrm{~b}$ & 1,38 & 37,77 \\
\hline & Diâmetro $(\mathrm{cm})^{(1)}$ & $0,84 \mathrm{a}$ & $0,83 \mathrm{a}$ & 0,01 & 2,16 \\
\hline \multirow{3}{*}{110} & $\mathrm{~N}^{\mathrm{o}}$ de folhas & $8,16 \mathrm{a}$ & $7,32 \mathrm{a}$ & 1,27 & 17,34 \\
\hline & Comprimento $(\mathrm{cm})$ & $6,29 \mathrm{a}$ & $8,43 \mathrm{a}$ & 2,18 & 31,34 \\
\hline & Diâmetro $(\mathrm{cm})^{(1)}$ & $0,89 \mathrm{a}$ & $0,86 \quad b$ & 0,01 & 2,15 \\
\hline \multirow{3}{*}{140} & $\mathrm{~N}^{\circ}$ de folhas & $14,44 \mathrm{a}$ & $11,10 \mathrm{~b}$ & 1,35 & 11,21 \\
\hline & Comprimento $(\mathrm{cm})$ & $21,81 \mathrm{a}$ & $12,47 \quad b$ & 3,38 & 20,85 \\
\hline & Diâmetro $(\mathrm{cm})^{(1)}$ & $0,93 \mathrm{a}$ & $0,90 \mathrm{~b}$ & 0,02 & 2,33 \\
\hline
\end{tabular}

Médias seguidas da mesma letra nas linhas não diferem entre si, pelo teste de Tukey, ao nível de 5\% de probabilidade.

${ }^{(1)}$ Médias transformadas em S.Q.R.T $(\mathrm{x}+\alpha) \alpha=0.5$.

o diâmetro. Aos 140 dias após a enxertia, as plantas submetidas ao vergamento apresentavam o comprimento da haste $75 \%$ maior do que as do tratamento por decapitação, o que possibilitaria a formação da muda em menor tempo, o que é de interesse do viveirista para maximizar a utilização do viveiro (Tabela 2). Esses resultados concordam com os obtidos em outros países. Embora a decapitação proporcione vantagem na brotação de borbulhas, o vergamento é o processo que mais estimula o desenvolvimento, considerando principalmente a altura da haste enxertada sobre citrumelo 'Swingle' (Bowman, 1999; Williamson \& Maust, 1996; Williamson et al., 1992).

A aplicação de cianamida hidrogenada não teve influência na brotação das borbulhas de laranja 'Valência' enxertada sobre citrumelo 'Swingle'.

A decapitação da haste do citrumelo 'Swingle' acima da enxertia acelerou a brotação de borbulhas de laranja 'Valência'. Por outro lado, o vergamento proporcionou maior vigor à brotação de laranja 'Valência', considerando o número de folhas, comprimento e diâmetro da haste.

\section{REFERÊNCIAS}

BARBASSO, D.V. Caracterização fenológica e físico-química de variedades de tangerinas em três porta-enxertos. 2005. 109f. Dissertação (Mestrado em Tecnologia da Produção Agrícola) Instituto Agronômico, Campinas, 2005.

BOWMAN, K.D. Comparison of two citrus bud-forcing methods for rapid propagation of scions on new hybrid citrumelo rootstocks. Hort Science, Orlando, v.34, n.1, p.42-143, 1999.

CAÑIZARES, A.; ROJAS, E. Efecto de la cianamida de hidrógeno y el ácido 2-cloroetil fosfónico sobre la foliación, floracin y fructificación de la lima 'tahiti'. Bioagro, Venezuela, v.13, n.1, p.10-14, 2001.

CARVALHO, E.F. Efeito do método de indução de brotação sobre o "pegamento e crescimento da haste do enxerto de laranja 'Pêra'. Pesquisa Agropecuária Brasileira, Braslia, v.27, n.3, p.473-478, 1992.

CARVALHO, S.A. Propagação dos citros. Informe Agropecuário, Belo Horizonte, v.22, n.209, p.21-25, 2001.

CASTRO, R.C.; MEDINA, C.L.; PACHECO A.C.; Potencialidade para a utilização de reguladores vegetais na citricultura brasileira. Laranja, Cordeirópolis, v.17, n.1, p.109-122, 1996.

COELHO, Y.S.; OLIVEIRA, A.A.R.; CALDAS, R.C. Efeitos do ácido giberélico $\left(\mathrm{GA}_{3}\right)$ no crescimento de porta-enxertos para citros. Pesquisa Agropecuária Brasileira, Brasília, v.18, p.1229-1232, 1983.

GIMENES-FERNANDES, N. \& BASSANEZI, R.B. Doença de causa desconhecida afeta pomares cítricos no norte de São Paulo e sul do Triângulo Mineiro. Summa Phytopathologica, Botucatu, v.27, p.93. 2001.

MARODIN, G.A.B.; SARTORI, I.A.; GUERRA, D. S. Efeito da aplicação de cianamida hidrogenada e óleo mineral na quebra de dormncia e produção de pessegueiro - 'Flamescre'. Revista Brasileira de
Fruticultura, Brasília, v.24, n.2.p.426-430, 2002.

MASHIMA, C. H.; HIRAI, R.D.; CAMARGO, U.A. Uva sem semente. Recife: SEBRAE-PE, 2000.53p.

MIELLE, A. Efeito da cianamida hidrogenada na quebra de dormência das gemas, produtividade do vinhedo e composição da uva Vitis vinifera L. cv. Cabernet Sauvignon. Pesquisa Agropecuária Brasileira, Braslia, v.26, n.3, p.315-354, 1991.

NORBERTO, P. M.; CHALFUN, N. N. J.; PASQUAL, M.; VEIGA, R. D.; MOTA, J.H. Efeito da época de poda, cianamida hidrogenada e irrigação na produção antecipada de figos verdes. Pesquisa Agropecuária Brasileira, Brasília, v.36, n.11, p.1363-1369, 2001.

OLIVEIRA, R. P.; SCIBITTARO, W.B.; BORGES, R.S.; NAKASU, B.H.; Muda de citros, 2005. Disponível em: $<$ http://www.cpact.embrapa.br/ sistemas/mudas/>. Acesso em: 02 maio 2005.

OMRAN, R. G. Peroxide Levels and the Activities of Catalase, Peroxidase, and Indoleacetic Acid Oxidase during and after Chilling Cucumber Seedlings. Plant Physiology, Washington, v.65, n.2, p.407-408, 1980

PÉREZ, F.J.; BURGOS, B. Alterations in the pattern of peroxidase isoenzymes and transient increases in its activity and in $\mathrm{H}_{2} \mathrm{O}_{2}$ levels take place during the dormancy cycle of grapevine buds: the effect of hydrogen cyanamide. Plant Growth Regultion, Dordrecht, v.43, n.3, p.213-220, 2004.

PETRI, J.L. Indução de brotação de gemas de macieira por cianamida hidrogenada e óleo mineral sob influência da temperatura. Pesquisa Agropecuária Brasileira, Brasília, v.32, n.1, p.71-75, 1997.

POMPEU JUNIOR, J. Cuidados no uso do Citrumelo Swingle. Revista Citricultura Atual, Cordeirópolis, v.3, n.13, p.7, 1999.

POMPEU JÚNIOR, J.; SALVA, R.; BLUMER, S. Copas e porta-enxertos nos viveiros de mudas cítricas do Estado de São Paulo. Laranja, Cordeirópolis, v.25, n.2, p.413-422, 2004.

SAMSON, J.A.; BINK, J.P.M. Citrus budding in the tropics: towards explanation of the favorable results of lopping. In: CONFERENCE OF THE INTERNATIONAL ORGANIZATION OF CITRUS VIROLOGISTS, 7., 1975, Atenas. Proceedings... Riverside: IOCV, 1976. p.213-231.

SCHAFER, G.; KOLLER, O. C.; SARTORI, I. A.; CASALI, M.E.; LIMA, J.G. Efeito de reguladores de crescimento, aplicados em diferentes épocas, e da incisão anelar dos ramos principais sobre a produção da laranjeira de umbigo 'Monte Parnaso'. Ciencia Rural, Santa Maria, v.31, n.4, p.577-581, 2001.

WILLIAMSON, J.G.; CASTLE, W.S.; KOCH, K.E. Growth and ${ }^{14} \mathrm{C}-$ photosynthase allocation in citrus nursery trees subjected to one of three bud-forcing methods. Journal of the American Society for Horticultural Science, Alexandria, v.117, n.1, p.37-40, 1992.

WILLIAMSON, J.G.; MAUST, B.E. Forcing treatment and rootstock effect budbreak and growth of containerized Citrus nursery trees. Hort Technology, Alexandria, v.6, n.2, p.134-137, 1996. 